rev.relac.int.estrateg.segur.10(2):69-87,2015

\title{
¿A QUIÉN ASEGURAMOS CUANDO HABLAMOS DE SEGURIDAD NACIONAL?: CONSIDERACIONES ONTOLÓGICAS SOBRE EL ESTADO-NACIÓN
}

\author{
Carlos Manuel Reyes Silva**
}

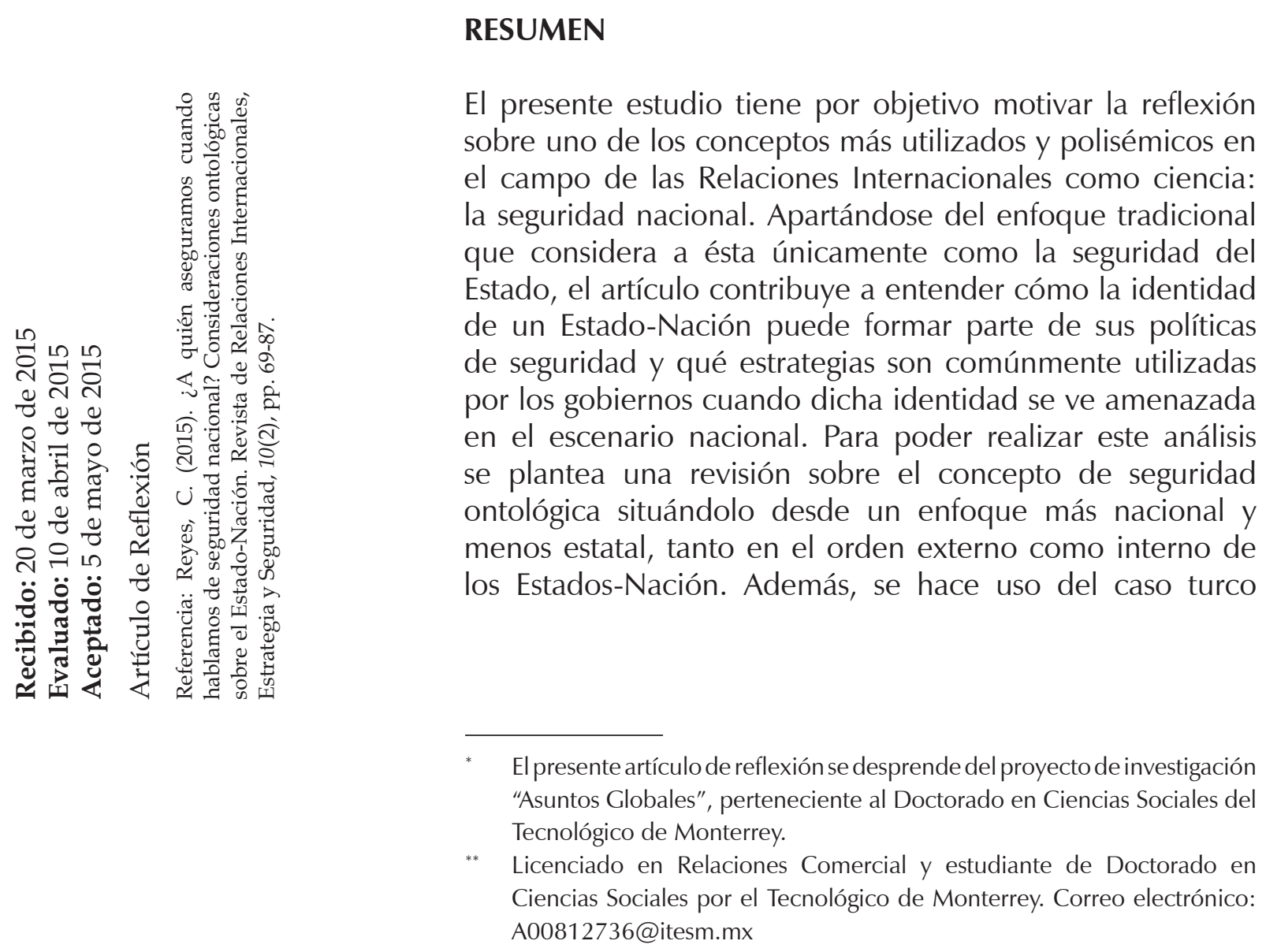


para ilustrar diversos fenómenos que versan sobre la importancia que tiene para los Estados el mantenimiento de sus identidades, aun en el actual sistema internacional.

Palabras clave: Ansiedad Ontológica, Articulación Hegemónica, Estado-Nación, Identidad, Narrativa, Seguridad, Seguridad Ontológica, Turquía

\title{
WHOM ARE WE REASSURING WHEN WE SPEAK OF NATIONAL SECURITY?: ONTOLOGICAL CONSIDERATIONS ABOUT THE NATION-STATE
}

\begin{abstract}
The aim of this article is to promote some reflections about a concept that is one of the most used and polysemic in the field of International Relation as a science: National Security. Getting away from the traditional approach which considers National Security as State Security, this article attempts to show how the identity of a Nation-state can -and should- be part of its security politics and to point out which strategies are commonly used by governments when those identities are threatened. In order to accomplish this analysis, it has been made an examination of the concept of Ontological Security, from a more national -and less statistapproach, as in the domestic level as in the external one. Additionally, the Turkish case has been used to illustrate some issues concerning to the importance that identities hold for the States, even in the current international system.
\end{abstract}

Keywords: Ontological Anxiety, Hegemonic Articulation, Nation-State, Identity, Narrative, Security, Ontological Security, Turkey

\section{A QUEM ASSEGURAMOS QUANDO FALAMOS DE SEGURANÇA NACIONAL?: CONSIDERAÇÕES ONTOLÓGICAS SOBRE O ESTADO-NAÇÃO}

\section{RESUMO}

O presente estudo tem por objetivo motivar a reflexão sobre um dos conceitos mais utilizados e polissêmicos no campo das Relações Internacionais como ciência: a segurança nacional. A parte da visão tradicional que considera a segurança nacional unicamente como a segurança do Estado, este artigo pretende ajudar a entender como a identidade de um Estado-Nação pode formar parte das políticas de segurança e que estratégias são geralmente utilizadas quando sua identidade é ameaçada. Para poder realizar esta análise, será necessária uma revisão sobre o conceito de segurança ontológica, começando deste um enfoque mais nacional e menos estatal, tanto na ordem interna como externa dos EstadosNação. Para, além disto, iremos recorrer do caso da Turquia para ilustrar empiricamente 
múltiplos fenómenos sobre a importância que tem para os Estados a manutenção da suas identidades, mesmo no atual sistema internacional.

Palavras-chave: Ansiedade Ontológica, Articulação Hegemônica, Estado-Nação, Identidade, Narrativa, Segurança, Segurança Ontológica, Turquia

\section{INTRODUCCIÓN}

Desde el establecimiento del Estado moderno, tras la firma del tratado de Westfalia en 1648, se ha desarrollado un ordenamiento internacional basado en sistemas de organización que detentan autoridad sobre ciertos límites territoriales y determinadas masas poblacionales. Con el advenimiento de otros eventos, este modo de organización buscó asentar su autoridad sobre la base de una legitimidad expresada en el lazo gobierno-sociedad, revestido a su vez con una figura nacional que ha servido para imaginar, construir y solidificar la identificación entre ambos.

A partir de esta misma lógica evolutiva es visible cómo en sus inicios la seguridad nacional referenciaba principalmente a la seguridad del Estado, su territorio y su población, sin ir más allá de un plano meramente físico; es decir, se aseguraban los condicionantes de continuidad de la sociedad pero no así el ethos de la comunidad. Esta relación ha permanecido casi incuestionable a lo largo de varias décadas, como queda reflejado en el concepto clásico de seguridad nacional; en este sentido, desde la perspectiva más tradicional que se desprende de algunos estudios de Relaciones Internacionales, se vincula a la seguridad nacional principalmente con las capacidades materiales de los Estados, su desarrollo militar, cuestiones geopolíticas y la latencia de múltiples amenazas.

Sin embargo, los actuales cuestionamientos que se adjudican tanto al papel que desempeña el Estado como a la vigencia de lo nacional, conllevan invariablemente una repercusión en lo que ocurre dentro del campo de la seguridad nacional. Es por ello que el objetivo del presente artículo es (re)construir el concepto de seguridad nacional a partir de la misma referencia del término -esto es la nación-, para diferenciarlo de los aspectos no identitarios del Estado. La principal finalidad de lo anterior es reconsiderar la relevancia que juega la cuestión de la identidad nacional en las relaciones internacionales a través del estudio de la seguridad y con ello, tener una mejor visión con respecto a las estrategias de las que hacen uso los gobiernos para afrontar las amenazas que se desprenden de este campo.

Con el afán de alcanzar dicho objetivo es necesario desarrollar una base teórica que de modo paralelo deconstruya y vuelva a construir la noción de seguridad nacional, para lo cual se hará uso de un concepto que ha sido recientemente popularizado en los estudios de corte constructivista: el de la seguridad ontológica. Por su parte, el componente empírico estará conformado por las constantes referencias a la República de Turquía ya que, debido a las distintas amenazas que ha enfrentado su seguridad ontológica -y también nacional stricto sensu-, se ha convertido en un caso casi paradigmático que vale la pena retomar para estos fines. 


\section{EL ENFOQUE TRADICIONAL DE LA SEGURIDAD}

La seguridad, por donde se mire, presenta una relación muy estrecha con el Estado. Lo anterior ocurre porque además de ser un modo de organización, los Estados modernos fueron previstos como un medio para limitar las agresiones externas; de hecho, el amplio control que tienen sobre los recursos y las armas les permite ser, incluso en la actualidad, el único actor capaz de asegurar físicamente a sus poblaciones frente a varias amenazas. Aunado a ello, la situación de anarquía en que se desarrollan las relaciones internacionales ha cimentado la importancia que se le ha dado a la seguridad, llegando a posicionarse como el fin supremo de los Estados (Waltz, 2010). Es por ello que dentro de este marco tradicional de pensamiento, una sociedad está y se presenta segura cuando el Estado ostenta cierto nivel de recursos y no presenta serias amenazas sobre ninguno de sus componentes.

Sin embargo, ¿De qué tipo de seguridad hablamos?, ¿Ello abarca la seguridad nacional o sería más preciso afirmar que estamos hablando de una seguridad societal, territorial o estatal?, ¿ No está encubriendo la seguridad nacional una mera seguridad física frente a diversas agresiones, ya sean internas o externas?, ¿Qué ocurre con lo nacional?, ¿Por qué se le denomina seguridad nacional a cuestiones de seguridad que si bien tienen una relación directa, no conforman propiamente ni coadyuvan al sostenimiento de lo nacional?, ¿Qué amenazas enfrenta lo nacional y cómo puede realizar un Estado una eficaz seguridad nacional? Además de los cuestionamientos anteriores, también podría agregarse que el concepto de seguridad nacional desde una perspectiva tradicional no termina por definir qué es el Estado, cuáles son sus límites o cuándo éste es fallido.

Por un lado, pudiera argumentarse que la calidad operativa de la que se desprende el uso de la seguridad nacional -desde esta perspectiva- recae en lo tangible de los peligros con base en su inclinación materialista, lo cual le ha permitido mantener su vigencia tanto en el lenguaje coloquial como en el académico; a modo de ejemplo baste señalar cómo el advenimiento de un ejército rival representa indudablemente una seria amenaza para la seguridad de la población y de los límites territoriales. Sin embargo, este ejemplo puesto bajo la luz de los eventos actuales queda sumamente restringido. Al respecto, la ampliación de la seguridad nacional -o meramente estatal, como hemos mencionado- a otros aspectos tales como la seguridad económico-comercial, ambiental o energética, evidencia la complejidad del concepto pero al mismo tiempo su propia limitación: la referencia a la protección de una sociedad en múltiples ámbitos, pero no así la seguridad de lo nacional, de lo comunitario o de su sentido identitario.

Es por ello que, partiendo de esta carencia, conviene introducir un cambio de enfoque que permita responder a estas críticas y cuestionamientos, aunque también se debe advertir que, como bien lo señala Mitzen (2006), la seguridad ontológica no es menos problemática que la física. No obstante, la importancia de rescatar la noción identitaria de la seguridad nacional reside en la forma en que ésta complementa al enfoque tradicional y contribuye a formular un panorama más integral, como quedará reflejado a continuación. 


\section{ASEGURAR EL ETHOS NACIONAL: LA SEGURIDAD ONTOLÓGICA}

A pesar de que el Estado y la nación representan fenómenos distintos, identificar dónde empieza y dónde termina la seguridad de cada uno resulta sumamente difícil de determinar. La fuerza del vínculo se desarrolla - partiendo de los supuestos más generales de ciertas teorías del nacionalismo, principalmente de los modernistas ${ }^{1}-$, un par de siglos después del establecimiento del sistema de Estados, cuando éstos se preocuparon por la legitimidad de su existencia y establecieron un sentido de identificación entre el Estado y el pueblo sobre el cual detentaban su autoridad.

Desde la situación señalada, Pérez Gil argumenta que "pese a las críticas ... la búsqueda de la supervivencia y seguridad del Estado promueve simultáneamente la seguridad de la sociedad o de la nación" (2012, p.209); si esto es cierto, entonces no debería de existir ninguna oposición al presentar dicha tesis en modo inverso, es decir, que la seguridad de la nación promueva la seguridad del Estado. Sin embargo, continuar en este camino de causalidades sería un objetivo que excede al del presente artículo por lo cual es sugerente referirse a ambos como mutuamente constitutivos -al menos en el actual orden internacional- y centrarse únicamente en el papel del Estado-Nación, como objeto de estudio, con respecto a la seguridad nacional.

Una vez aclarado lo anterior, el cuestionamiento acerca de qué es la seguridad nacional debe empezar por tratar de responder a quién se está asegurando o quién es esa nación a la que se le debe proteger. Al respecto, ésta supone la existencia -ya sea real, retórica o imaginariade una nación que, en términos muy generales, implica una colectividad cohesionada de modo horizontal (un sentido comunitario) por una cultura, una historia y un territorio con el que presenta cierta identificación. Ahora, para ayudar a entender cómo se relaciona lo nacional con las cuestiones de seguridad -lo que en un sentido lógico-estructuralista del lenguaje sería la seguridad nacional- es recomendable tomar en consideración otro término: la seguridad ontológica.

Al respecto, la "seguridad ontológica" es un término de reciente uso en las Relaciones Internacionales que se desprende del trabajo de Anthony Giddens (1995) para hacer referencia -desde un plano microsocial- al enfrentamiento del sujeto con un entorno prolífico en riesgos e incertidumbre, el cual denomina como modernidad tardía. De acuerdo a Giddens, "ser ontológicamente seguro es poseer en el nivel del inconsciente y de la conciencia práctica,

1. Al respecto, la obra de Ernest Gellner (2008) sobresale como una respuesta a este proceso. Sin embargo, otras corrientes del nacionalismo tales como el constructivismo o el poscolonialismo también pueden compartir en lo general este supuesto de una construcción descendente del nacionalismo (de las élites hacia las masas) como un medio de legitimación y cohesión social. En este sentido, el análisis que realiza Özkirimli (2010) sobre las teorías del nacionalismo es revelador porque permite detectar este tipo de similitudes entre las teorías y subrayar una base discretamente consensual. 
respuestas a cuestiones existenciales fundamentales que se plantea de alguna manera toda vida humana" (1995, p.66); por lo que la identidad -en este caso nacional- forma parte de estos cuestionamientos existenciales aun si se traslada del individuo al Estado-Nación.

En este punto es posible prever el surgimiento de ciertas críticas en contra de dicha extrapolación, por lo que es necesario detenerse y profundizar en esta cuestión, misma que será aprovechada para introducir otro elemento a considerar: la narrativa. Primeramente se debe mencionar que el análisis de la seguridad ontológica propuesto, al menos en este ensayo, no parte de una perspectiva esencialista sino crítica; en este sentido, pretende introducir un nuevo enfoque para problematizar uno de los conceptos más comunes pero más desvirtuados en el campo de las Relaciones Internacionales. En segundo lugar, la denominada 'antropomorfización' del EstadoNación -que en realidad simplemente muestra al Estado como un agente colectivo- es una herramienta heurística que coadyuva a un mejor entendimiento de este fenómeno; además, el hecho de que los Estados-Nación actúen como si fueran agentes o unidades homogéneas, al menos en el terreno discursivo, justifica su uso en este caso. Finalmente, la tercera justificación afirma que la extrapolación de la seguridad ontológica del individuo al Estado-Nación es viable porque en ambos casos hay un sentido reflexivo de sí mismos mediante la figura de la narrativa.

Asimismo, la narrativa del Estado-Nación tiene una capacidad representacional y performativa en sus ciudadanos como cualquier acto del habla², lo cual a su vez da lugar a tres fenómenos: en primer lugar, la narrativa permite la unicidad de la historia del Estado-Nación, o lo que es lo mismo, un desplazamiento temporal coherente de sí mismo; al respecto, Ringmar (retomado en Berenskoetter, 2012) afirma que la ventaja de la narrativa es que permite aprehender el desenvolvimiento de la vida del agente de una forma no estática, de modo que el desplazamiento no sacrifica su unidad y tampoco su dinamismo. En segundo lugar, la narrativa posibilita una interpretación de lo doméstico y de lo externo, esto es que permite la interpretación, descripción e inteligibilidad de la realidad dotándola de significados (Berenskoetter, 2012). Por último, la narrativa dará ocasión para vincular ciertas políticas con dicha interpretación del mundo en un intento de convertirse en un medio de justificación (Steele, 2008). Por ello, de acuerdo a los fenómenos anteriores es factible afirmar que si bien Giddens proponía que la seguridad ontológica era el "sentimiento de continuidad y orden en los sucesos" (1995, p.295), la narrativa es el proceso de anclaje que permite su posibilidad.

Así, como acertadamente señala Steele, "sin una narrativa ... el self de un Estado no existe" (2008, p.20), y en consecuencia, la idea de una nación que refleja el ethos comunitario sería automáticamente desechada. Bajo esta lógica, otros académicos como Subotic y Zarakol (2013) apoyan la idea de que los Estados-Nación tienen una memoria y cuentan historias acerca de sí

2. Para profundizar el papel del lenguaje en los estudios de Relaciones Internacionales y su capacidad performativa, véase Onuf (2013). 
mismos, de modo que sin éstas no existiría la figura del self, ni en su modalidad particular ni en la colectiva. Cabe mencionar que esta memoria es selectiva y dejará fuera ciertos sucesos para subrayar otros de acuerdo a los intereses de ese momento, es decir, habrá algunas experiencias más significantes que otras (Berenskoetter, 2012), así como algunas que obedezcan a determinados intereses y ello requiera el sacrificio o la omisión de otras. Igualmente se debe agregar que la narrativa va a situar al Estado-Nación no sólo en un espacio determinado sino en un eje temporal, el cual va desde lo experimentado hasta lo deseado ${ }^{3}$ para mantener un cierto grado de seguridad ontológica, ya que se debe recordar que la pretensión de ésta es limitar lo incierto, dentro del cual el futuro juega un papel fundamental.

Por ello, todos los Estados-Nación construyen continuamente una narrativa e incluso cabe la posibilidad de encontrar varias narrativas al interior de cada uno, pero ello será explicado en el apartado destinado al orden interno. Por lo pronto, es momento de observar cómo los Estados-Nación construyen y defienden su seguridad ontológica; para ello, se comenzará por el orden externo o internacional para, posteriormente, analizar la misma problemática en el orden interno o doméstico.

\section{EL MEDIO INTERNACIONAL: INTERACCIONISMO Y ANSIEDAD ONTOLÓGICA}

Como se mencionó, los Estados-Nación no presentan únicamente necesidades materiales sino que también manifiestan necesidades identitarias, por lo que ambas requieren de enfoques de seguridad distintos aunque tampoco excluyentes. Así, a pesar de que los Estados existen por procesos de construcción internos, necesitan de un reconocimiento por parte del Otro para existir totalmente en un sentido ontológico ${ }^{4}$ (existir como un Estado-Nación, un ser EstadoNación en el mundo) y en un sentido óntico ${ }^{5}$ (con las particularidades propias de ser un EstadoNación, diferenciado). Así, de modo casi paradójico, el Estado-Nación tiene una disposición de presentarse como un agente diferenciado pero a su vez con una condición similar a la de

3. Véase el artículo de Berenskoetter (2012) para comprender la relación de los horizontes espacio-temporales con la narrativa.

4. Es evidente que el reconocimiento, en nuestro orden internacional, es un factor fundamental. En los casos de ciertas entidades que buscan autonomía con miras a conformar un Estado-Nación, lo primero que buscan es el reconocimiento por parte de otro Estado o alguna Organización Intergubernamental que les respalde y les acredite como tal ante la comunidad internacional.

5. En este sentido, lo ontológico y lo óntico no han sido estudiados como procesos diferenciados en la literatura especializada. Sin embargo, la diferencia entre ambos puede ser el origen para justificar el uso del término "seguridad óntica", la cual sí estaría directamente vinculada con la identidad nacional del Estado. Al respecto, es como si lo ontológico se relacionara únicamente con un sentido de "ser en el mundo" mientras que lo óntico con el "modo particular de ser en el mundo". Por lo pronto se continuará haciendo uso del término "seguridad ontológica", implicando dentro de ésta a la otra. 
Ios demás; esto, al parecer, responde también a una necesidad doméstica, ya que de acuerdo a Mitzen (2006), los Estados deben buscar su seguridad ontológica para proveer la seguridad ontológica de sus miembros.

Es por ello que la noción ontológica debiera considerarse un eje fundamental para los estudios sobre seguridad, tanto por ser una cuestión que trata de responder a qué se refiere la "seguridad nacional" como por el hecho de que las cuestiones identitarias proveen los contextos necesarios para interpretar las amenazas en el escenario internacional. Desgraciadamente, para que pueda existir una verdadera preocupación por analizar este tipo de seguridad se requiere la presencia de determinados peligros, pues en caso contrario el interés en ésta se vería reducido, particularmente por el pragmatismo imperante en el campo de la política exterior.

Así, se puede comenzar reflexionando sobre qué sucede cuando el Estado-Nación ve amenazada su seguridad ontológica de modo general. Para ello, hay que volver nuevamente al concepto de seguridad ontológica de Giddens (1995), especialmente cuando señala que la seguridad en el mundo depende de la confianza en los demás, lo que la vuelve intrínsecamente relacional; de este modo, un Estado no tiene la palabra final respecto a este tipo de seguridad porque depende de los Otros (Mitzen, 2006). Chantal Mouffe (2011), hace esta misma referencia al rol que juegan los Otros como una precondición de la existencia de la identidad del actor, la cual denomina "exterioridad constitutiva"; en el caso de los Estados-Nación, el resto de Estados son un ejemplo de ese elemento externo que constituye parcialmente la identidad y la seguridad del actor en cuestión.

En consecuencia, la seguridad del Estado-Nación puede verse constituida pero a la vez amenazada por algún Otro, ya sea interno o externo, real o imaginario. Aquí habría que señalar también que no todos los Otros tienen el mismo peso, por lo cual es útil recurrir al concepto de "otros significantes", los cuales, de acuerdo a Wendt (1999), son aquellos que ejercen mayor influencia sobre el actor en cuestión. Para poder introducir las referencias al caso turco, cabe señalar que no todos los Otros representan lo mismo para la identidad y la seguridad ontológica de dicha nación: al interior, los kurdos han sido durante décadas el otro significante, mucho más si se les compara con otros grupos étnicos como los circasianos o incluso frente a varios grupos religiosos. Por su parte, países tales como Alemania, Armenia, Azerbaiyán, Chipre, Francia, Grecia, Irán y Rusia también son mucho más significativos para su identidad que algún Estado latinoamericano e incluso que muchos de Medio Oriente.

El papel de los Otros como una amenaza a la seguridad ontológica es mucho más visible cuando ellos no responden a lo que estaba previsto. En este sentido, un agente con un grado aceptable de seguridad ontológica confía -o suspende la $d u_{d}{ }^{6}-$ en que los demás seguirán actuando

6. Giddens (1995) afirma que un agente no puede vivir en un estado constante de incertidumbre. Esta situación hace referencia al concepto "epojé de la actitud natural" de Alfred Schutz (2008), que a diferencia de la "epojé 
de la misma manera, de modo que el primero puede canalizar su energía en la persecución de objetivos en vez de enfocarla hacia la manutención de lo rutinario (Mitzen, 2006). Sin embargo, esta situación no es siempre prolongable; a menudo se originan cambios en la respuesta de otros Estados que activan la incertidumbre. A modo de ejemplo cabe señalar que durante gran parte de la Guerra Fría, Turquía tenía un grado aceptable de seguridad ontológica, particularmente cuando se visualizaba como parte de la Comunidad Europea; así, podía darse el lujo de suspender esa duda mientras perseguía otros objetivos particulares. La situación cambió drásticamente cuando, tras los eventos que siguieron a la caída del muro de Berlín, los países de la Comunidad se preocuparon por integrar a los Estados de Europa Central y del Este, postergando la inclusión de Ankara a pesar de haber solicitado su adhesión con antelación. Consecuentemente, su seguridad ontológica de sentirse y presentarse como un Estado europeo era minada, del mismo modo que lo fue su sentido de pertenencia a Occidente en la lucha contra el comunismo.

Así, durante los casos en que se produce una pérdida en la predictibilidad, el Estado puede verse desorientado -con intereses quizá confusos-, desubicado e inmerso en un entorno cognitivo nuevo en el cuál no sabe cómo debe de actuar. También puede ocurrir que, partiendo de que la seguridad ontológica del Estado satisface aquella de sus miembros (Mitzen, 2006), una pérdida en la del primero repercuta en la de estos últimos. En ambos casos se presenta el mismo fenómeno, denominado "ansiedad ontológica", la cual puede traducirse en la experiencia de una identidad que no encuentra un reconocimiento en el Otro. Al respecto, se le ha denominado ansiedad para diferenciarla del miedo, el cual está relacionado a una amenaza hacia la supervivencia -propio del enfoque tradicional o físico de seguridad-, mientras que la primera tiene que ver con una amenaza a la identidad (Steele, 2008). Esta situación, en el caso turco, parece ser la constante más que la excepción, principalmente por la imagen que ha construido y que, a pesar de todos los esfuerzos, no ha sido reconocida del mismo modo por sus contrapartes europeas.

Otro fenómeno que puede poner en riesgo la seguridad ontológica es la disonancia ontológica. Dicho concepto, de acuerdo a Amir Lupovici (2012), hace referencia al choque que se presenta entre la gama de soluciones destinadas a resolver una amenaza identitaria, es decir, no sólo involucra un conflicto entre las identidades del Estado-Nación -lo cual sería, de acuerdo al mismo autor, una disonancia identitaria-, sino entre las estrategias que sirven para salvaguardar cada una de éstas. Al respecto, para poder tener una mejor interpretación de estos eventos, a continuación se profundizará en algunas de las principales opciones que tienen los Estados para proteger su seguridad ontológica

fenomenológica", pone la duda misma entre paréntesis o la suspende. Esta suspensión se prolongará hasta el arribo de alguna irrupción que obligue al actor a revisar sus creencias. 


\section{SEGURIDAD NACIONAL: ESTRATEGIAS QUE PARTEN DE LA IDENTIDAD}

Para hacer frente a las amenazas descritas en el apartado anterior, los gobiernos pueden hacer uso de ciertas técnicas. En primer lugar, como se vio, el Estado-Nación salvaguarda constantemente su seguridad ontológica mediante la rutinización (Mitzen, 2006; Steele, 2008). De este modo, la repetición de ciertas prácticas tales como comportamientos y discursos, la institucionalización de acuerdos, el mantenimiento de relaciones -tanto de amistad como de enemistad-, suponen una reducción en el ámbito de lo incierto. Incluso si el Otro no respondiera del modo previsto a la identidad del Estado-Nación, la repetición misma logrará que, eventualmente, se le sea reconocido como tal; este proceso es lo que genera el alter-casting ${ }^{7}$.

Sin embargo, cómo también se señaló, existe una serie de irrupciones que pueden acabar con la rutinización establecida; en palabras de Giddens, "Ios momentos decisivos amenazan la coraza protectora que defiende la seguridad ontológica del individuo, pues quiebran la actitud de dejar que las cosas sigan como están" (1995, p.147). Es en estos casos cuando el establecimiento de rutinas ha dejado de dar resultados para reducir la incertidumbre del entorno o, simplemente, no generan la respuesta esperada por parte del Otro, obligando al actor a buscar otra vía de acción.

Al respecto, una de las principales soluciones es la evasión. Lupovici (2012) plantea esta estrategia porque, en concordancia con la teoría de Giddens, dicha acción ayuda a excluir determinadas situaciones o al menos, neutralizarlas. Así, se supone que un incremento en la ambigüedad permite posponer una situación en la cual no se sabe cómo actuar, lo cual posibilita la espera de una nueva respuesta o de nuevos llamados de interpelación por parte de los Otros para establecer un entorno cognitivo. De acuerdo a este autor, la evasión es una opción atractiva porque no involucra un cambio identitario y de hecho, él mismo explica como la Unión Europea ha preferido la evasión ante la candidatura turca porque de ese modo no sacrifica su seguridad ontológica -y recuérdese que tampoco la óntica-. Lo mismo sucede para Turquía, quien tampoco ha decidido institucionalizar o participar en otro proceso de integración sino que ha preferido continuar con un rol ambivalente tanto en relación con los europeos como con los países árabes.

Una opción distinta a la evasión es la representación de sí mismo como un ente único, la cual propone Demirtaş Bagdonas (2012) con el afán de resaltar la diferenciación respecto de los demás. En su análisis, ella señala cómo Turquía ha asegurado cierto estatus, principalmente en relación a Europa, por las cartas de presentación que ha utilizado en el medio internacional,

7. De acuerdo a Wendt (1999), durante esta situación un Estado actúa en un modo particular para tratar de inducir en el Otro un tipo de comportamiento esperado. De ese modo, se puede señalar que el Estado tratará al Otro de modo que éste se relacione con el primero en la forma deseada, construyendo así un tipo particular de situación. 
tales como "puente entre civilizaciones" o un Estado "multi-identitario". En este tipo de casos lo que se busca es resaltar alguna característica de diferenciación -suponiendo que esto le dotará de mayor valor- pero que de modo paralelo, promueva la similitud con los Otros, ganándose su respeto y recuperando o fortaleciendo su seguridad ontológica.

También se puede controlar la ansiedad ontológica (re)identificando a los amigos de los enemigos externos o de aquellos internos pero que son extraños o ajenos al Estado-Nación. En estos casos hay una evolución que va de una situación de ansiedad a un entorno donde el miedo -como amenaza a la seguridad física- es identificable; esta situación es lo que Huysmans (1998) denomina "construcción de amenazas" y la cual provee de un entorno menos incierto para el actor. Para continuar con la extrapolación al caso turco, la situación de los kurdos en dicho Estado se vuelve ejemplar. Si bien durante décadas esta comunidad coadyuvó a formar un grado de ansiedad para el gobierno turco, la formación del Partido de los Trabajadores del Kurdistán (PKK) y su correspondiente contingente paramilitar, permitieron que la ansiedad ontológica se convirtiera en una amenaza física. En consecuencia, la visión de la sociedad se transformó para dar cabida a una creciente cohesión, ya que el fenómeno era más visible e identificable, lo cual aclaraba el entorno cognitivo. Con base en lo anterior es viable afirmar que, a pesar de que la relación con el Otro es amenazante, al mismo tiempo es esencial para la seguridad ontológica del Estado-Nación, tanto que de acuerdo a Huysmans (1998), existen daños irreparables dentro de una comunidad cuando la otredad se diluye. En este mismo sentido se debe recordar el señalamiento de Chantal Mouffe (2011) respecto a la esencia de lo político, misma que radica en la participación de colectividades con identidades e intereses irreconciliables, donde la figura del Otro es un elemento imperdible.

Otra estrategia sumamente interesante es que cuando la seguridad ontológica choca con la física, los Estados-Nación pueden preferir resguardar la primera aun con los costes que puede implicar para la segunda. Así, de acuerdo a Mitzen (2006) los Estados-Nación pueden involucrarse en un conflicto o perpetuarlo antes de dañar su propia identidad, ya que una situación alternativa supondría repensar otras estrategias para sopesar la ansiedad ontológica. Para ilustrar esta cuestión es importante recordar el involucramiento de Turquía en la guerra de Corea (1950-1953), mismo que le permitió reducir su ansiedad y además, ayudó a su correspondiente inclusión en la Organización del Tratado del Atlántico Norte, brindándole la seguridad ontológica necesaria al garantizarle un asiento entre los países de Occidente, lo cual representaba la materialización del sueño ataturquista. Lo mismo puede señalarse de su involucramiento en la Guerra del Golfo (1990-1991), en su intento de intervención en Siria (2013) y en su actual participación en la lucha contra el Estado Islámico de Iraq y el Levante (EIIL). En este sentido, los ejemplos anteriores confirman que el Estado turco prefiere arriesgar parcialmente su seguridad física para reducir la ansiedad ante su potencial desplazamiento por parte de los países europeos y de Estados Unidos.

Por último, dentro de este apartado, se debe señalar que las amenazas a la seguridad ontológica no siempre resultan perjudiciales. Al respecto, hay académicos como Steele (2008) que señalan 
que la ansiedad es necesaria para salir adelante o como Mitzen (2006), quien asevera que la flexibilidad ante la incertidumbre permite un mayor aprendizaje que la rigidez, de modo que quienes puedan distanciarse de sus rutinas tendrán una mayor capacidad de aprendizaje. Incluso si la ansiedad es producto de la humillación, ésta no es del todo negativa para la seguridad ontológica; al respecto, Krolikowski (2008) señala que dicho discurso solidifica el sentido de pertenencia a un grupo.

Siguiendo dicha idea, Subotic y Zarakol (2013) introducen el concepto de la "intimidad cultural" en este campo, el cual consiste en todos aquellos aspectos de una cultura que pueden avergonzarla pero que paralelamente provee a los que la comparten de una identidad común; en palabras de las autoras, "lo que sostiene la identidad del Estado es también aquello que está expuesto al criticismo internacional" (Subotic \& Zarakol, 2013, p.932). Respecto al caso turco es posible encontrar elementos de este estilo, ya sean históricos -como la situación armenia, el caso de Chipre o la opresión de los kurdos-, culturales -entre los cuales puede servir de ejemplo la religión musulmana después de los eventos del 11 de septiembre- o tradicionales - como una base social patriarcal con tintes machistas-. Al final, aunque esta serie de situaciones les exponen a la crítica frente a los Otros, ejercen igualmente una función importante en su sentido nacional.

\section{EL PROCESO INTERNO: ARTICULACIÓN Y LUCHA HEGEMÓNICA}

La construcción y el mantenimiento de la identidad del Estado-Nación y la consecuente seguridad ontológica no ocurren en su totalidad por una interacción con otros actores; también se requiere de un proceso interno, tal como existía en la división teórica de la persona propuesta por Mead (1973) -con la figura del "yo" y el "mí" - o en la primera distinción que hacía Alexander Wendt de ambas para el caso de los Estados ${ }^{8}$-diferenciando la identidad corporativa de la identidad social- (Wendt, en Lapid \& Kratochwil, 1996).

A pesar de las constantes críticas por dejar el "yo" del Estado-Nación en un nivel prácticamente desatendido (Berenskoetter, 2012; Hopf, 2002; Hudson, 2001; Zehfuss, 2001), el énfasis en la incidencia de la interacción con otros actores sobre la identidad del Estado se debe en gran medida a la renuencia de las Relaciones Internacionales por involucrarse en los fenómenos meramente domésticos. Por ello, es importante romper con dicha tradición y presentar una

8. Si bien los primeros artículos de Wendt sólo separaban estos dos tipos de identidades, el autor fue modificando su teoría. Al respecto, en su obra de 1999, Alexander Wendt ya presentaba una tipología de cuatro identidades: corporativa o individual, tipo, rol y social. Sin embargo, igual que hicieron Cederman y Daase (2006), se agruparán a las últimas tres como parte de las identidades sociales para poder diferenciarlas de la corporativa, siendo la única que ocurre completamente en un ámbito doméstico. 
serie de consideraciones que ocurren en este nivel, para lo cual se exigen algunos cambios en el enfoque, sobre todo para trasladarlo de un proceso interaccionista a otro que permita observar la construcción, el sostenimiento y la defensa del ethos comunitario en un sentido más autárquico.

Si bien es cierto que los fenómenos del entorno externo repercuten directamente en la construcción interna de la narrativa del Estado-Nación ${ }^{9}$-como por ejemplo, la figura del Otro externo-, es importante observar que en el nivel doméstico se produce un juego de poder entre varias colectividades. Así, como bien señala Bozdağlioğlu (2003), en sociedades multiculturales existe una lucha entre varios grupos, cada uno presionando para que sus concepciones identitarias se vean reflejadas en la identidad nacional, llegando en casos extremos a suprimir aquellos referentes que no favorezcan la homogeneización de la población. En este mismo sentido se expresaba Bloom (1993) al subrayar la competencia intergrupal que busca apropiarse de la identidad nacional con miras a legitimarse como su verdadero protector.

En realidad, el señalamiento que hacen ambos autores no es tan reciente, ni como fenómeno ni en su teorización. Para ello es conveniente rescatar la obra de Gramsci, porque de ahí se desprenderá el origen del enfoque que se propone retomar para estudiar el orden interno. Al respecto, Gramsci (2005) había advertido que el posicionamiento de un grupo en el gobierno no podía bastarse únicamente con el respaldo del poder político ni de la dominación por medio del capital, sino que era necesario desarrollar modelos ideológicos que legitimaran al grupo en cuestión, esto es la hegemonía cultural.

Sin embargo, es conveniente alejarse momentáneamente de la noción hegemónica para ir un paso más atrás, esto es hacia el proceso de articulación. En este sentido, si la noción de hegemonía se introdujera desde el inicio, la articulación se entendería desde un sistema de relaciones de poder -el cual, si bien es evidente que existe en el campo político, no necesariamente tiene que fluir de las élites hacia las masas-. Por ello, al desplazar la cuestión hegemónica, es posible aprehender la articulación en su modo más elemental como un proceso, más allá de los agentes que intervienen en él y de sus intenciones, evitando así las ataduras quasi ideológicas.

Para ello, la articulación de la identidad nacional desde un enfoque procesual puede ser explicada tomando como base la obra de Ernesto Laclau (2011a; 2011b; \& Mouffe, 1987), de la cual sólo se recuperarán los componentes más relevantes para el caso que aquí ocupa.

9. Al respecto, uno de los esfuerzos integradores más prometedores ha sido el de Xavier Guillaume (2011), quien parte de un enfoque dialógico para demostrar los vicios epistemológicos que se desprenden de separar los procesos interno y externo de construcción y sostenimiento de la identidad. Tomando como base los planteamientos del dialogismo de Mikael Bakhtin, Guillaume resalta las propiedades extrínsecas de las características internas de la identidad y cómo son endogeneizadas. 
Partiendo de esta aclaración, Laclau explica que la generación e interpelación de identidades populares amplias -entre las cuales se encuentra obviamente la nación- tiene su base en el lenguaje, pero no tanto por los actos del habla sino por la cuestión de los significantes vacíos. Al respecto, un significante vacío es aquél que no tiene un significado anclado al mismo, siendo dicha carencia conceptual lo que le permite operar como un punto de identificación, constituyendo y representando una comunidad.

Así, se puede comenzar suponiendo una comunidad conformada por diversos grupos, cada uno de las cuales tiene ciertas demandas, mismas que pueden entenderse desde una perspectiva identitaria como demandas de reconocimiento o simplemente como demandas de comportamiento, de identificación o de "dar un sentido al mundo", es decir, como demandas de seguridad ontológica si es que ese término pudiera ser válido. A su vez, dichas demandas tienen una capacidad particularizante -propia de cada grupo-, y otra que les permite ser equivalentes entre sí frente a un mismo opuesto, siendo este componente compartido el que permite articular una relación equivalencial entre ellas.

Consecuentemente, el elemento que representa el vínculo equivalencial de las demandas es el que tomará el lugar del significante vacío -la nación- y el cual, consecuentemente, dotará de significado a la vacuidad que ocupaba ese lugar. A este proceso Laclau lo denomina como hegemonía, la cual es "una relación por la cual una cierta particularidad pasa a ser el nombre de una universalidad que le es enteramente inconmensurable" (2011a, p.15). También es importante mencionar que durante todo este fenómeno de articulación ocurre un proceso paralelo de exclusión, ya que existirán demandas que no puedan ser totalmente integradas a la cadena equivalencial, las cuales pueden denominarse como proyectos insubordinados, o como les designa Laclau, proyectos hegemónicos rivales.

De este modo, Laclau advierte que la construcción de una identidad popular -léase nuevamente nacional-, conlleva inherentemente una encarnación de lo universal en lo particular (2011a), el cual incluso forma parte de la misma representación: "La identificación con un significante vacío es la condición sine qua non de la emergencia de un pueblo ... el significante vacío es algo más que la imagen de una totalidad preexistente: es lo que constituye esa totalidad" (Laclau, 2011b, p.204).

El proceso recién descrito, que devela el aspecto hegemónico que ocurre en la construcción de estas identidades, es esclarecedor en tanto que permite la aprehensión y el estudio de las identidades sin recurrir a nociones esencialistas.

Ahora, en lo que a este artículo concierne, no sería errado suponer y proponer que la articulación y la noción hegemónica de una identidad nacional, contribuyen a la seguridad ontológica $-y$ todavía más a la óntica- del Estado-Nación. Tampoco sería desacertado afirmar que aquellos proyectos alternativos, o incluso contrahegemónicos, son capaces de generar ansiedad 
ontológica en el actor en cuestión; así, aunque haya una identidad nacional predominante o hegemónica, ésta siempre podrá ser puesta a prueba o cuestionada por algún agente interno (Parekh, 2008). De este modo, se conserva lo expuesto en la teoría de la seguridad ontológica, misma que como se mencionó, descansa siempre sobre una base relacional.

Cabe agregar que la ansiedad ontológica no solamente puede ser provocada por estos proyectos excluidos que se tornan rivales sino que puede originarse al interior del proceso mismo. Para ello se debe tener en cuenta que una mayor extensión en la cadena equivalencial conducirá a una mayor heterogeneidad en su composición, lo cual es significativo, ya que a medida que la cadena es más extendida, es menos factible que se produzca una clara identificación de lo externo -el Otro-, que puede ser interno en términos de ubicación física. Además, dicha heterogeneidad puede desestabilizar la cadena e incluso puede dar pie a que exista alguna contradicción al interior de la misma.

Para ejemplificar el proceso de articulación, téngase en cuenta nuevamente el caso de Turquía. A partir de la llegada de Atatürk al poder, no hubo un proceso de articulación hegemónica sino de imposición de acuerdo a Laclau (2011b). Aun así, este proceso que podría denominarse "articulación impuesta" solventó durante mucho tiempo la seguridad ontológica turca con elementos europeos tanto en la cultura -una nueva moda-, en la religión -la laicidad-, en el lenguaje -la purificación del turco-otomano y la adopción del alfabeto latino-, y en la relación con el Otro -Europa como proyecto de asimilación y los países árabes como amenaza-. Este proyecto se tornaría posteriormente hegemónico con la existencia de otros programas de nación que estuvieron latentes durante gran parte del siglo veinte, los cuales, en cualquier intento de cobrar fuerza, fueron reducidos por el estamento militar y el resto de la élite kemalista. Sin embargo, la llegada del Partido de la Justicia y el Desarrollo (AKP, por sus siglas en turco) en 2002, supuso un nuevo proceso de articulación hegemónica. El AKP conformó una amplia cadena de equivalencia entre las demandas identitarias de la comunidad, misma que se ha ajustado con el paso de los años; así, esta estrechez se puede observar con el paso de un primer momento representado en una Turquía "multi-identitaria" e "incluyente" hacia un segundo periodo donde el Islam figura más en el discurso y en las acciones oficiales, de modo que muchas colectividades internas han sido nuevamente desplazadas de la concepción nacional.

Ahora bien, así como quedó establecido en el nivel externo, el orden interno también presenta algunas de las mismas estrategias para salvaguardar la seguridad ontológica. Por un lado, dicha seguridad se protege nuevamente mediante la rutinización, principalmente por medio de los actos del habla que se generan en el discurso oficial, así como en las fiestas nacionales, los monumentos, la educación y los medios de comunicación. Lo mismo ocurre con otros mecanismos ya vistos tales como la evasión e igualmente, con la conversión de la ansiedad en miedo mediante la identificación de amenazas físicas, siendo los inmigrantes los sujetos más vulnerables. Sin embargo, el proceso que principalmente reconfigura y constantemente 
salvaguarda la seguridad ontológica a nivel interno ocurre mediante la articulación de la lógica equivalencial, misma que tratará siempre de representar a una sociedad homogénea y que ésta se sienta identificada con las interpelaciones del Estado ${ }^{10}$.

\section{CONCLUSIÓN}

Los Estados-Nación están preocupados por generar y sostener una identidad colectiva, en parte porque de ella se deriva la unidad de la sociedad, la cual, homogeneizada a través de dicho ideal de nación, deviene en una de las principales fuentes de legitimidad del primero. Además, de acuerdo al orden internacional en el que vivimos, estos actores deben representarse ante la comunidad con un estatus similar -el Estado- pero con ciertas particularidades -la nación-.

Las situaciones anteriores generan una preocupación por mantener una seguridad ontológica y óntica, que les permita a los Estados-Nación definirse a sí mismos, sus intereses, y su entorno, siendo esto lo que debiera referir el carácter nacional del concepto de seguridad nacional. Sin embargo, éste se ha vuelto tan vacío como los significantes a los que se hacía referencia, lo cual no es un asunto menor ya que como bien señala Pérez Gil, "el problema radica en que si se dan muchos significados, el concepto de seguridad nacional se torna inmanejable" (2012, p.203), tal como ha venido sucediendo con la inclusión de cada vez más factores que pretenden atender a las problemáticas actuales.

Así, el concepto de seguridad nacional ha sido reservado para atender únicamente cuestiones físicas, desplazando este otro tipo de preocupaciones que enfrentan los Estados-Nación y que, en sentido estricto del término, son las que refieren a asegurar lo nacional. Cabe mencionar que lo estipulado en este artículo no pretende abogar por un retorno a los nacionalismos agresivos, mismos que produjeron en su tiempo conflictos inolvidables para la humanidad; al contrario, invita a entender la forma en que se mantiene y reproduce lo nacional, incluso en un entorno tan cambiante como el actual y con una abundancia cada vez mayor en cuanto a los referentes para las identidades de los individuos.

Otra cuestión a subrayar es que el concepto de seguridad nacional puede ser reinterpretado en esta otra forma -ontológica y óntica- para hacerle justicia a su denominación y sobretodo, para entender una serie de actitudes de los Estados-Nación en cuanto a la conformación de sus narrativas, su capacidad de movilización e interpelación, la interpretación que realiza de las amenazas (Lupovici, 2012) y la estabilidad de sus relaciones sociales, tanto cooperativas como

10. Vale la pena retomar aquí el señalamiento de Kinvall (2004) sobre la capacidad de la nación para mostrar una imagen de seguridad o de un hogar libre de intrusos, lo cual en tiempos de crisis o de grandes cuestionamientos, funge como uno de los principales anclajes identitarios del individuo. 
conflictivas (Mitzen, 2006). Además, este nuevo enfoque ha permitido vincular dos niveles que, aun mediante procesos distintos, conforman el mismo fenómeno de la identidad nacional, lo cual es una superación casi epistemológica respecto a los estudios que se decantan por un solo nivel o de aquellos que los conciben de manera independiente.

Ahora bien, está por demás aclarar la existencia otros factores ligados a la seguridad nacional que van a coadyuvar al sostenimiento de ésta en su sentido ontológico. Al respecto, es importante no perder de vista que las demandas que conforman una cadena equivalencial no sólo son satisfechas con el mero reconocimiento sino que existe un componente material; así, como acertadamente sostiene Beatriz Sarlo: "cuando ser argentino no significa ni trabajo, ni comida, ni tiempo, vale poco ser argentino. La nacionalidad no es sólo imaginaria. Se arraiga en su inscripción material sobre los cuerpos" (2010, p. 18). Sin embargo, la importancia de éste y otros componentes ha sido escasamente analizada, por lo que se recomienda su posible inclusión y profundización en futuros estudios al respecto.

Por último también es interesante señalar que, pese a las serias amenazas que enfrentan cotidianamente los Estados latinoamericanos - particularmente con la aparición de grupos que cuestionan la legitimidad del Estado y pretenden fungir como nuevos polos de interpelación-, el enfoque de la seguridad ontológica no haya cobrado su correspondiente importancia en los estudios producidos en esta región. Aun así merece la pena ahondar en el tópico no sólo para esta zona geográfica, ya que el paradigma estatocentrista de las Relaciones Internacionales se ve desafiado por el fortalecimiento de otros actores en el medio internacional y por la múltiple proliferación de referentes identitarios propia de la posmodernidad. Este panorama exige, en consecuencia, que los Estados sean más conscientes de quiénes son (su existencia óntica, el ethos nacional), cuáles son sus intereses, cómo deben de actuar y, sobretodo, a quién están asegurando cuando redactan y ejecutan sus políticas de seguridad.

\section{REFERENCIAS}

Berenskoetter, F. (2014). Parameters of a national biography. European Journal of International Relations, 20 (1), 262-288. doi: 10.1177/1354066112445290

Bloom, W. (1993). Personal identity, national identity and international relations. Gran Bretaña: Cambridge University Press.

Bozdağlioğlu, Y. (2003). Turkish foreign policy and Turkish identity: a constructivist approach. Gran Bretaña: Routledge Series.

Cederman, L. \& Daase, C. (2006). Engogeneizing corporate identities: the next step in Constructivist IR Theory. En Guzzini, S. \& Leander, A. (Eds.), Constructivism and International Relations: Alexander Wendt and his critics (pp. 117-137). Nueva York: Routledge. 
Demirtaş, Ö. (2012). A Shift of Axis in Turkish Foreign Policy or A Marketing Strategy?: Turkey's Uses of Its 'Uniqueness' vis-à-vis the West/Europe. Turkish Journal of Politics, 3(2), 111-132.

Gellner, E. (2008). Naciones y nacionalismo. Madrid: Alianza Editorial.

Giddens, A. (1995). Modernidad e identidad del yo: el yo y la sociedad en la época contemporánea. Barcelona: Península.

Guillaume, X. (2011). International Relations and Identity: A dialogical approach. Nueva York: Routledge Series.

Gramsci, A. (2005). Antología: Selección, Traducción y Notas de Manuel Sacristán. México: Siglo $\mathrm{XXI}$.

Hopf, T. (2002). Social Construction of International Politics: Identities and Foreign Policies. Estados Unidos: Cornell University Press.

Hudson, V. M. (2007). Foreign Policy Analysis: Classic and Contemporary Theory. Estados Unidos: Rowman \& Littlefield Publishers.

Huysmans, J. (1998). Security! What Do You Mean?: From Concept to Thick Signifier. European Journal of International Relations, 4(2), pp. 226-255. doi: 10.1177/1354066198004002004

Kinvall, C. (2004). Globalization and Religious Nationalism: Self, Identity, and the Search for Ontological Security. Political Psychology, 25 (5), pp. 741-767. doi: 10.1111/j.14679221.2004.00396.x

Krolikowski, A. (2008). State Personhood in Ontological Security Theories of International Relations and Chinese Nationalism: A Sceptical View. Chinese Journal of International Politics, 2, 109-133. doi:10.1093/cjip/pon003

Laclau, E. \& Mouffe, C. (1987). Hegemonía y estrategia socialista: hacia una radicalización de la democracia. Madrid: Siglo XXI.

Laclau, E. (2011a). Debates y combates: Por un nuevo horizonte de la política. Buenos Aires: Fondo de Cultura Económica.

(2011b). La razón populista. Buenos Aires: Fondo de Cultura Económica.

Lupovici, A. (2012). Ontological dissonance, clashing identities, and Israel's unilateral steps towards the Palestinians. Review of International Studies, 38(4), 809-833. doi: 10.1017/ S0260210511000222 
Mead, G. (1973). Espíritu, persona y sociedad: desde el punto de vista del conductismo social. Barcelona: Paidós.

Mitzen, J. (2006). Ontological Security in World Politics: State Identity and the Security Dilemma. European Journal of International Relations, 12(3), 341-370. doi: $10.1177 / 1354066106067346$

Mouffe, C. (2011). En torno a lo político. Buenos Aires: Fondo de Cultura Económica.

Onuf, N. (2013). Making Sense, Making Worlds: Constructivism in social theory and international relations. Gran Bretaña: Routledge.

Özkirimli, U. (2010). Theories of Nationalism: A Critical Introduction. Nueva York: Palgrave Macmillan.

Parekh, B. (2008). A new politics of Identity: Political Principles for an Interdependent World. China: Palgrave Macmillan.

Pérez, L. (2012). Elementos para una teoría de la política exterior. México D.F.: Tirant lo Blanch.

Sarlo, B. (2010). Tiempo presente: notas sobre el cambio de una cultura. Buenos Aires: Siglo XXI.

Schutz, A. (2008). El problema de la realidad social: Escritos I. Buenos Aires: Amorrortu.

Steele, B. (2008). Ontological security in International Relations: self identity and IR state. Nueva York: Routledge.

Subotic, J., \& Zarakol, A. (2013). Cultural intimacy in International Relations. European Journal of International Relations, 19(4), 915-938. doi: 10.1177/1354066112437771

Waltz, K. (2010). Theory of International Politics. Nueva York: Waveland.

Wendt, A. (1996). Identity and Structural Change in International Politics. En Lapid, Y. \& Kratochwil, F. (Eds.), The Return of Culture and Identity in IR Theory (pp. 47-63). Estados Unidos: Lynne Rienner Publishers.

Wendt, A. (1999). Social Theory of International Politics. Reino Unido: Cambridge University Press.

Zarakol, A. (2010). Ontological (In)security and State Denial of Historical Crimes: Turkey and Japan. International Relations, 24(1), 3-23. doi: 10.1177/0047117809359040

Zehfuss, M. (2001). Constructivism and Identity: A dangerous liaison. European Journal of International Relations, 7(3), 315-348. doi: 10.1177/1354066101007003002. 